\title{
Time Perspective as A Common Transdiagnostic Factor of Mental Problems: A Systematic Review and Meta-Analysis Protocol
}

\section{Yi Yang ( $\sim$ s2060087@ed.ac.uk)}

The University of Edinburgh https://orcid.org/0000-0002-3238-5748

\section{Siu Ching Wong}

The University of Edinburgh

Ingrid Obsuth

The University of Edinburgh

Aja Murray

The University of Edinburgh

\section{Protocol}

Keywords: Time perspective, Temporal Focus, Mental health, Transdiagnostic, Systematic Review, MetaAnalysis

Posted Date: March 31st, 2021

DOI: https://doi.org/10.21203/rs.3.rs-353702/v1

License: (c) (i) This work is licensed under a Creative Commons Attribution 4.0 International License. Read Full License 


\section{Abstract}

\section{Background}

Research into the transdiagnostic processes underlying multiple mental health problems is promising for making clinical practice and interventions more effective and resource-efficient. In this protocol, we describe a systematic review and meta-analysis that will explore time perspective, defined as an individual's relative investment of attention on past, present, and future, as a possible transdiagnostic factor that may contribute to issues across wide-ranging domains of mental health.

Methods

A systematic review and meta-analysis will be conducted to quantify the associations between mental health issue and specific dimensions of time perspective (past, present, future), respectively. The review will include quantitatively measured associations between time perspective and psychological problems published in a peer-reviewed journal from $1^{\text {st }}$ January 1990 up until $1^{\text {st }}$ March 2021, in the English language. Electronic searches will be conducted in Google Scholar, PubMed, MEDLINE, PsycINFO, and Web of Science, and EMBASE and supplemented by expert consultation and inspection of the reference lists of included papers. Screening, quality assessment and data extraction will be conducted by two reviewers independently, and potential conflicts will be resolved through discussion with a third reviewer. Random-effects meta-analysis will be conducted using the metafor package in R statistical software, and quality assessment will employ The Newcastle-Ottawa Quality Assessment Form for Cohort Studies and for Case-Control Studies. The Quality In Prognosis Studies (QUIPS) will be used to evaluate risk of bias. A narrative synthesis will additionally be used to summarize and interpret the results.

Discussion

The present review will provide the first systematic synthesis exploring the associations between time perspective defined as a multidimensional construct and a broad range of mental health issues. This will help evaluate the extent to which time perspective can be considered a key transdiagnostic factor in mental health and thus a key intervention target for the prevention and treatment of multiple mental health issues simultaneously. With a clearer view of the relations between time perspective and various mental health issues based on a robust synthesis, more focused, effective, and efficient interventions may be delivered.

Systematic review registration

PROSPERO CRD42021228869

\section{Background}

\section{Statement of the Problem}


For decades, the focus of clinical diagnosis has been on classifying mental health problems into separate disease categories, as can be observed in the various versions of the International Classification of Diseases (ICD) and Diagnostic and Statistical Manual (DSM). However, the prevalence of comorbidity, or co-occurrence, among ICD or DSM disorders has cast doubt on the idea that these classifications reflect topographic variations $(1-4)$. Further, the distinct diagnostic categories defined in frameworks such as the DSM are inconsistent with the findings from clinical neuroscience, which indicates redundancies among classifications and suggests that they are not capturing the common underlying syndromes (5). Based on these findings, a new transdiagnostic trend is developing, which aims to identify shared underlying mechanisms across different classes of mental health problems, to provide more parsimonious classification systems, and identify treatment techniques targeting common underlying mechanisms $(3,6,7)$. Research into transdiagnostic processes underlying multiple mental health problems is, therefore, promising for making clinical practice and interventions more effective and resource-efficient. In this protocol, we describe a systematic review and meta-analysis that will explore time perspective as a possible transdiagnostic factor that may contribute to issues across wide-ranging domains of mental health.

\section{Conceptualization of Time perspective}

Time perspective, sometimes referred as "temporal focus", is defined as an individual's relative investment of attention on the past, present and future and is assumed to filter temporal information and influences behaviour (8-10). Zimbardo and Boyd (11) described time perspective as a nonconscious personal attitude, which helps to give order, coherence, and meaning to the continual flow of existence. Importantly, it assigns life experiences into temporal categories. In other words, time perspective is like lens through which one's life events are processed (12). A particularly influential theory on time perspective has been Zimbardo's theory, which proposes that time perspective is situationally determined, relatively stable, and subject to individual differences. Based on this definition, Zimbardo and colleagues have operationalised time perspective and developed a widely used and well-validated assessment tool, Zimbardo Time perspective Inventory (ZTPI) $(11,13)$. ZTPI consists of 56 items which are scored on a 5point Likert scale ranging from 1-very uncharacteristic to 5-very characteristic. Based on a mix of cognitive, affective and behavioural items, the ZTPI yields 5 dimensions of time perspective: pastnegative (aversive view of the past), past-positive (warm, sentimental attitude toward the past), presenthedonistic (enjoyment, pleasure risk-taking attitude towards life), present-fatalistic (helpless and hopeless attitude towards the future and life), and future (planning for and achievement of future goals) (11). Zimbardo's theory also emphasizes a balanced time perspective, which is defined as the mental ability to switch flexibly among the different time perspectives based on the situation and personal resource (11, 12). Other assessment tools have also been constructed to evaluate a comprehensive time perspective view, such as the Temporal Orientation Scale (TOS) (14), the Balanced Time perspective Scale (BTPS) including past and future $(15,16)$, and the Temporal Focus Scale (TFS) with past, present and future included (10). These instruments have, however, been less widely used than the ZTPI (e.g., according to google scholar citations until 6th March 2021, ZTPI: TOS: BTPS: TFS = 3986:485:118:507). 
Growing evidence illustrates that people organise their life experience using the frame of time as a reference which shapes their judgements, decisions and behaviours (8), as well as mentally constructs the continuity and stability of self, others and objects (17). However, the relations between time perspective and psychological problems are not straightforward. Overemphasizing any one frame (past, present or future) could cause bias and bring dysfunctions (11). For example, low future and high present temporal orientation is a key underlying process in impulsive decision-making regardless of future consequences (18), or choosing "the more immediate smaller rewards over the later bigger rewards" in delay discounting (19), which contributes to impulsivity related mental problems (20,21). Also, despite future orientation is a predictor of well-being and achievement (22-24), being biased towards the future does not correlate with subjective happiness due to less enjoyment of the present pleasure and goalrelated stress $(23,25)$. It is for this reason that the ability to flexibly switching between time frames is proposed as an indicator of mental health $(12,26,27)$, and a balanced time perspective is deemed to consist of high past-positive, moderate future, moderate present-hedonistic, low past-negative and low present-fatalistic time orientation (28). A balanced time perspective has been linked to general satisfaction with life $(29,30)$, while biasing to a particular time orientation is potentially a predictor of different problematic human behaviours (31).

\section{Time perspective as An Important Underlying Element in Intervention Techniques}

Previous research suggests that time perspective is related to a range of psychological traits and processes that have been implicated in the risk for different types of mental health issues. For example, time perspective has been linked to impulsivity (important in issues such as attention deficit hyperactivity disorder, ADHD) and addiction (32,33), emotional dysfunction (important in issues such as mood disorders) (34), and identity discontinuity (important in issues such as personality disorders) (35). As such, a time perspective may be promising for illuminating, profiling, and addressing a broad range of mental health issues $(17,26,36,37)$. For example, the ZTPI could be a potential diagnostic tool, such as the present hedonism scale for impulsivity related mental problems (32). Further, an assessment of how patients organize and feel about their past, present and future experiences can be a starting point for planning intervention; and high past-positive and moderate future time perspectives can be the index for subject well-being (28).

Indeed, there is already some evidence for the clinical utility of time perspective as an intervention target. A recent systematic review by Rung and Madden (37), analysing the effectiveness of intervention techniques in reducing impulsive choice (bias to choose the more immediate rewards despite it is smaller than the long-term one) on delay discounting, showed that time perspective, as encoded in concepts such as "temporal priming" (experimentally manipulating participants' perception of time), "framing of time" (description of intertemporal choices with equivalent outcomes across different time frames), and "episodic future thinking" ( imagining or episodic simulation of one's future) was the key effective element in numerous intervention studies. Time perspective is, in fact, an existing element in many psychological therapies (38). One well-evidenced transdiagnostically effective therapy, acceptance and 
commitment therapy (ACT), includes time perspective elements. For example, the Value intervention element of ACT encourages people to look into their long-term values with future positive outcomes while the "contact with the present moment" and "self as context" components, which are adopted from the mindfulness skill sets, train people to focus on the ongoing flows of the present moment $(7,39,40)$.

Further, based on Zimbardo's theory, Time perspective Therapy, aimed at restoring a balanced time perspective to shift from the past to present and to future, was developed at the beginning in treating mental problems that "stuck in the painful past", such as post-traumatic stress disorder (PTSD), anxiety and depression symptoms (41-43). Despite limited evidence so far, Time perspective Therapy is regarded as promising in treating difficulties associated with ADHD and impulsivity (32), particularly in conjunction with established therapies (44-46). Finally, one study suggested that targeting selfdiscontinuity, a sense that the present self is fundamentally different from the past self, was effective in changing addiction behaviours among problem gamblers and problem drinkers by serving as a catalyst in moving people from addiction to positive action (47).

\section{Rationale for A Systematic Review}

Despite previous studies identifying links between time perspective and mental health issues and preliminary evidence that it is a potentially useful intervention target, no comprehensive overview of the links between specific time perspectives and different mental health issues is available to guide the development and targeting of therapeutic components to optimize their effectiveness. Robust evidence on this is critical for mapping time perspective interventions to specific problems, including considering time perspective as a potential transdiagnostic intervention target that can be used in prevention and intervention efforts for a wide range of mental health issues simultaneously. A systematic review and meta-analytic study on the link between time perspective and related psychological problems is thus valuable for offering a robust foundation for clinical techniques targeting time perspective.

Despite the value of considering a more encompassing multidimensional construct of time perspective, previous studies have disproportionately exclusively focused on future time perspective and there have been a number of systematic reviews on this topic. For instance, Henry and colleagues (22) reviewed the future time perspective in occupational contexts, examining its antecedents and consequences in 33 primary studies, and found that both occupational future time perspective (OFTP) and future time perspective (FTP) were related to occupational well-being, motivation, attitude and behaviour. Rudolph and colleagues (48) conducted a meta-analysis of 40 primary studies on the antecedents and outcomes of OFTP, and the results showed that OFTP was related to important work outcomes (job satisfaction, organizational commitment, work engagement, retirement intention), work continuance intentions and task and contextual performance (49). Andre and colleagues (50) reviewed the predictive power of FTP in education, work and health domains of 77 studies, and found that the strength varied for attitudes, perceived behaviour control, behaviour intention and behaviours. Kooij and colleagues (23) analysed 212 studies regarding FTP across 65 years (1950-2015), and demonstrated that FTP was positively correlated with grade point average, life satisfaction, subjective health, physical exercise, and retirementrelated financial knowledge, while negatively related with anxiety, depression, risk behaviour and 
substance abuse. They also reported that FTP was associated with outcomes over and above the effects of personality traits, showing its incremental contribution over and above major dispositional factors. All these findings indicated that time perspective may play an important role in a wide range of domains of people's life; however, they exclusively focused on the future time perspective or analysed its relations with various life domains but not including mental health issues. Recent research found that people scoring high on future dimension do not necessarily score low on the present or past dimensions, demonstrating the need to consider a multidimensional view of time perspective $(9,23)$ and suggesting that reviews focusing only on future perspective may be missing a key part of the picture.

Taken together, there remains a need for a systematic synthesis of the evidence for time perspectivemental health associations based on a time perspective conceptualisation that includes a focus on the past, present and future. A recent systematic review study helped shift attention to the relation between Balanced Time perspective/Deviation from the Balanced Time perspective and psychological variables (well-being, mental health, personality, cognitive functioning, self-control, interpersonal relations, biological features, and demographic variables), and concluded from 49 studies that BTP in general were an important mechanism of adaptation, with particularly pronounced effect on well-being (12). However, this study, together with the previously mentioned reviews, did not discuss different dimensions of time perspective and their relationship with various mental health issues from a transdiagnostic angle to provide a 'bigger picture' view of whether and how time perspective may contribute to a range of mental health issues. This is not due to a lack of primary studies: when a scoping search (using the terms showed in the "search strategy" section of the present study) in titles and abstracts have been conducted in PubMed to gain an overview of the range and depth of the present topic, a total number of 1076 articles were located. Therefore, the current protocol describes a systematic review and meta-analysis of the association between the different dimension (past, present, future) of time perspective and various mental health issues of the existing literature.

\section{Review Questions}

- What mental problems correlate/are predicted by each specific dimension of time perspective (past, present, future), respectively?

\section{Methods}

The present study followed the recommendations from the Preferred Reporting Items for Systematic Reviews and Meta-Analysis Protocols (PRISRMA-P) to prepare and report the systematic review and meta-analysis protocol. The present study has been preregistered on PROSPERO (The International Prospective Register of Systematic Reviews), with registration number CRD42021228869.

\section{Search Strategy}

Four information sources/strategies will be combined to search for studies analysing the association between time perspective and psychological problems: 
- Online computer database searches, including PubMed, OVID MEDLINE, EMBASE, PsycINFO, and Web of Science;

- Searches for relevant studies in the citations of other time perspective related systematic reviews and meta-analyses;

- Forward and backward citation chaining of included key papers and author's publications;

- Expert consultations with academics in the field of time perspective research.

Search terms related to time perspective and psychological problems $(10,12,33)$ will be combined with Boolean operations. The following terms will be searched in the title, abstracts, and keywords. The number of papers retrieved from each database will be recorded. The search strategy will be customized for each database but the terms will include:

- ("time perspective" OR "perspective of time" OR "temporal perspective" OR "time focus" OR "temporal focus" OR "time altitude" OR "time perception" OR "time horizon" OR "time orientation" OR "temporal orientation" OR "past focus" OR "future focus" OR "present focus" OR "past orientation" OR "future orientation" OR "present orientation" ) AND

- ( "mental health" OR psychopathology OR "psychological health" OR "mental problem" OR "mental illness" OR "psychological problem" OR "mental abnormality" OR "psychological abnormality" OR disorder OR dysfunction OR "substance use" OR "substance abuse" OR drinking OR alcohol OR smoking OR "drug abuse" OR "drug use" OR addiction OR gambling OR "binge eating" OR bulimia OR anorexia OR mania OR compulsivity OR compulsive OR impulsivity OR impulsive OR aggression OR aggressive OR self-injury OR suicide OR "conduct disorder" OR "oppositional defiant disorder" OR schizophrenia OR "schizophrenia spectrum" OR anxiety OR "generalized anxiety disorder" OR "separation anxiety" OR anxious OR depression OR depressive OR mood OR bipolar OR panic OR borderline OR histrionic OR antisocial OR narcissistic OR paranoid OR schizoid OR Schizotypal OR "avoidant personality" OR "dependence personality" OR "obsessive-compulsive personality" OR "cluster A" OR "cluster B" OR "cluster C" OR psychosis OR "personality disorder" OR PTSD OR "Posttraumatic stress disorder" OR trauma OR "hyperkinetic disorder" OR ADHD OR "attention deficit disorder" OR "attention deficit hyperactivity disorder" OR autism OR ASD OR ASC OR "autism spectrum disorder" OR "autism spectrum conditions" ) NOT

- (medicine OR medical OR medicines OR pharmacy OR pharmaceutical)

\section{Inclusion Criteria}

- Type of studies: quantitatively measured the association between time perspective and psychological problems by an odds ratio (OR), a correlation coefficient, or regression coefficient, or with data to calculate these statistics.

- The time frame and publication status: Published in a peer-reviewed journal from 1st January 1990 up until 1st March 2021;

- Language: available in the English language; 
- Setting: no specific requirement;

- Evaluation of time perspective: multiple dimensions or a single dimension of time perspective (past, present, future);

- Multi-dimensional time perspective should use ZTPI, ZTPI Short Form, BTPS, TOS, and the TFS as the measurement; or other multidimensional measures of good validity and reliability;

- Single dimensional time perspective should use the Occupational Future Time perspective Scale (OFTPS) (51), the Consideration of Future Consequences Scale (CFCS) (52), the Future Time perspective Scale (C\&L, unpublished manuscript; Carstensen LL \& Lang FR, 1996;Stanford University) (22, 53), Future Time perspective scale (H\&M) (54), Future Time Orientation Scale (55), or other single dimension time perspective scales;

- Study Design: both cross-sectional and longitudinal quantitative studies.

\section{Exclusion Criteria}

- Studies that evaluated intervention effects using time perspective related therapy;

- Studies that calculated the relationship between the antecedent factors and time perspective;

- Studies that measured time perspective with free-response measures and open-ended questionnaires;

- Studies achieve low-quality scores in the quality assessment;

- Studies that don't have sufficient information to calculate an effect size.

\section{Data Extraction and Management}

Zotero reference management software will be used to extract and manage the references, which will also be used to remove the duplicate references. A data extraction form will be designed to extract information from the included studies. The extraction will include the following information:

- Study Characteristics: study; location; author; year; title;

- Methodology/Data Collection: study design; data source; sample size; age of participants; types of psychological problem; the measurement tool for time perspective; the included dimensions of time perspective; Cross-sectional and longitudinal studies; with control group or not;

- Data selection: time perspective variables; regression, correlation and other methods; other predictors;

- Results (point $\mathrm{R}^{2}, \mathrm{~F}, \beta, r, p$, odd ratio, and others), if in the process of review any new categories are identified, they will be added to the data extraction.

A pilot test, including database search, publication selection and quality assessment, will be conducted to test the screening instructions on 5 random studies until 100\% agreement is achieved between reviewers, to ensure the consistency. During screening process, two reviewers will independently screen titles and 
abstracts. Following this, full text articles will be obtained and reviewed by the two reviewers independently using the data extraction form. A third reviewer will discuss with the two independent reviewers to make a final inclusion when disagreements emerge between the two reviewers on the inclusion/exclusion studies.

\section{Quality Assessment and Risk of Bias}

The PRISMA checklist will be used to evaluate the quality of the selection. There are several versions of the Newcastle-Ottawa Scales (NOS) for different study designs. The present study will adopt the NOS quality assessment for cohort studies and the NOS for case-control studies. The Newcastle-Ottawa Quality Assessment Form for Cohort Studies will be used to assess three domains for participants selection ( 6 stars), comparability of study groups ( 2 stars), and outcome ( 5 stars). A study can be given a maximum of one star for each numbered item within the Selection and Outcome categories. A maximum of two stars can be given for Comparability. The standard for good quality is 3 or 4 stars in the selection domain, 1 or 2 stars in the comparability domain, and 2 or 3 stars in the outcome domain. Fair quality equals 2 stars in the selection domain, 1 or 2 stars in the comparability domain, and 2 or 3 stars in the outcome domain. The Newcastle-Ottawa Quality Assessment Form for Case-Control Studies will assess selections (4 items with 4 stars), comparability (1 item with 2 stars), and explosion (3 items with 4 stars). A study can be given a maximum of one star for each numbered item within the Selection and Exposure categories. A maximum of two stars can be given for Comparability. Besides, the Quality In Prognosis Studies (QUIPS) will be employed to evaluate the risk of bias in predictor findings. It guides reviewers thinking and judgment about the overall risk of bias within each of the 6 domains: study participation, study attribution, prognostic factor measurement, outcome measurement, study confounding, statistical analysis and reporting. Each domain is judged on a three-grade scale (low, moderate or high risk of bias). If the overall methodology of high quality (+++), it means the majority of criteria met, little or no risk of bias, and the results unlikely to be changed by further research. If the overall methodology is acceptable $(++)$, it means most criteria met, some flaws in the study with an associated risk of bias, and conclusions may change in the light of further studies. Low quality $(+)$ means either most criteria not met or significant flaws relating to key aspects of study design.

\section{Data Synthesis}

The R software metafor Package (56) will be used to conduct the meta-analysis of the data extracted from the included studies. In order to estimate the standardized effect, a meta-analysis will be applied. The statistics include the followings:

- Meta-analysis: all statistical results will be converted to a common effect size metric where possible (correlation coefficient for continuous outcomes and odds ratios for dichotomous outcomes) for meta-analysis.

- Cross-sectional and longitudinal studies will be analysed separately, as will adjusted and unadjusted findings; 
- A meta-analysis will be conducted for past, present, future outcome measure across studies separately to determine their predictive power to a certain range of mental problems, respectively;

- Heterogeneity test with $\mathrm{I}^{2}$ and the Q-statistic.

- Moderators will be added as predictors to the random-effects model to test moderation of the association by factors for which sufficient information will be available, such as age group of the sample (adults, adolescents, children), publication date, study location, and methodological quality score, nature of the sample (e.g., clinical, normative forensic).

- If quantitative synthesis cannot be warranted, a narrative review will be carried out.

\section{Assessment of Reporting Bias}

- A funnel plot will be employed to test the asymmetry of outcome measures from the publications using the Egger test and the Begg and Mazumdar's rank correlation test;

- The trim and fill method will also be adopted to identify publication bias and assess its likely impact on results.

\section{Discussion}

The present study protocol presents the plans for a systematic review and meta-analysis of the associations between time perspective and mental health issues. Previous reviews have predominantly focused on reviewing the future time perspective despite growing acceptance that time perspective is best conceptualized as a multidimensional construct. Further, those reviews have not addressed the correlations between mental health problems from a multidimensional time perspective, which is important because different dimensions of time perspective may map to risks for different types of problems (e.g., emotional problems, impulsivity, personality disorders).

Such a review can provide critical insights for developing and targeting intervention methods to base on altering maladaptive or rigid time perspectives. A systematic review can offer the following benefits to support these efforts: It can provide a more robust estimate of the time perspective-mental health associations than any primary study; it can evaluate whether the field may have been affected by publication bias; it can identify whether there are gaps in the evidence that can be addressed in future research, e.g., specific mental problems that have received only scant attention in this area; it can provide an assessment of the methodological quality of studies in the field to gauge how reliable the evidence is and make recommendations for how to improve the quality of future studies; and it can look for moderators, for example, whether the strength of the association is stronger for adolescence or adulthood, thus helping with the targeting of interventions to different stages of development. With a clearer view of the relations between time perspectives and mental health issues, more effective and better-targeted interventions can be facilitated.

\section{Limitations}

Page 10/18 
It is important to consider the limitations of the review. First, because historically, a large number of studies have focused on the future time perspective, studies on the present or the past time perspective are of a relatively small number, which may reduce confidence in the accuracy of the aggregate effect sizes observed for these constructs. Second, since the present review is limited to the English language articles in major databases and from citations of relevant studies and expert consultation, the studies published in non-English languages will be ignored, which may bring potential reporting bias. Third, there are variations of the time perspective definition regarding the measures used in different studies, which may bring potential heterogeneity between studies. Finally, we will focus on published peer-reviewed studies because this ensures that studies have been subject to a degree of quality control. This means that though we will analyse publication bias using the studies we retrieve and we cannot be certain of the extent to which publication bias is present.

\section{Conclusion}

A systematic review and meta-analysis of the association between different types of time perspectives and a wide range of mental health issues will be valuable for informing transdiagnostic approaches to preventing and treating mental health issues. The review will allow us to provide more robust estimates of the associations between time perspective dimensions and mental health, examine the moderators of these associations, and characterize the strength of the evidence in this area as well as identify specific methodological weaknesses and evidence gaps that can be addressed in future studies. This can help support efforts to develop and improve mental health interventions with maladaptive time perspectives as a target.

\section{Abbreviations}

Acceptance and commitment therapy, ACT

Attention deficit hyperactivity disorder, ADHD

Balanced Time perspective Scale, BTPS

Consideration of Future Consequences Scale, CFCS

Diagnostic and Statistical Manual, DSM

Future time perspective, FTP

International Classification of Diseases, ICD

Newcastle-Ottawa Scales, NOS

Occupational future time perspective, OFTP 
Occupational Future Time perspective Scale, OFTPS

Odds ratio, $\mathrm{OR}$

Preferred Reporting Items for Systematic Reviews and Meta-Analysis Protocols, PRISRMA-P

Quality In Prognosis Studies, QUIPS

Temporal Focus Scale, TFS

Temporal Orientation Scale, TOS

Zimbardo Time perspective Inventory, ZTPI

\section{Declarations}

Ethics approval and consent to participate

N/A.

Consent for publication

N/A.

Availability of data and materials

N/A.

Competing interests

The authors declare that they have no competing interests.

\section{Funding}

N/A.

\section{Authors' contributions}

Y.Y. conceived the study and took the lead on drafting the manuscript; S.C.W., I.O. and A.L.M. provided critical feedback on drafts and contributed to drafting the manuscript. All authors read and approved the final manuscript.

Acknowledgements

N/A. 


\section{References}

1. Bickel WK, Mueller ET. Toward the Study of Trans-Disease Processes: A Novel Approach With Special Reference to the Study of Co-morbidity. J Dual Diagn. 2009;5(2):131-8.

2. Brown TA, Barlow DH. A Proposal for a Dimensional Classification System Based on the Shared Features of the DSM-IV Anxiety and Mood Disorders: Implications for Assessment and Treatment. Psychol Assess. 2009;21(3):256-71.

3. Sauer-Zavala S, Gutner CA, Farchione TJ, Boettcher HT, Bullis JR, Barlow DH. Current Definitions of "Transdiagnostic" in Treatment Development: A Search for Consensus. Behav Ther. 2017;48(1):12838.

4. Sharma V, Coleman S, Nixon J, Sharples L, Hamilton-Shield J, Rutter H, et al. A systematic review and meta-analysis estimating the population prevalence of comorbidities in children and adolescents aged 5 to 18 years. Obes Rev. 2019;20(10):1341-9.

5. Insel T, Cuthbert B, Garvey M, Heinssen R, Pine DS, Quinn K, et al. Research Domain Criteria (RDoC): Toward a New Classification Framework for Research on Mental Disorders. Am J Psychiatry. 2010;167(7):748-51.

6. Bickel WK, Jarmolowicz DP, Mueller ET, Koffarnus MN, Gatchalian KM. Excessive discounting of delayed reinforcers as a trans-disease process contributing to addiction and other disease-related vulnerabilities: Emerging evidence. Pharmacol Ther Oxf. 2012;134(3):287-97.

7. Levin ME, Haeger J, Ong CW, Twohig MP. An Examination of the Transdiagnostic Role of Delay Discounting in Psychological Inflexibility and Mental Health Problems. Psychol Rec. 2018;68(2):201-10.

8. Keough KA, Zimbardo PG, Boyd JN. Who's Smoking, Drinking, and Using Drugs? Time perspective as a Predictor of Substance Use. Basic Appl Soc Psychol. 1999;21(2):149-64.

9. Mohammed S, Marhefka JT. How have we, do we, and will we measure time perspective? A review of methodological and measurement issues. J Organ Behav. 2019;41(3):276-93.

10. Shipp AJ, Edwards JR, Lambert LS. Conceptualization and measurement of temporal focus: The subjective experience of the past, present, and future. Organ Behav Hum Decis Process. 2009;110(1):1-22.

11. Zimbardo PG, Boyd JN. Putting time in perspective: A valid, reliable individual-differences metric. J Pers Soc Psychol. 1999;77(6):1271-88.

12. Stolarski M, Zajenkowski M, Jankowski KS, Szymaniak K. Deviation from the balanced time perspective: A systematic review of empirical relationships with psychological variables. Personal Individ Differ. 2020;156:109772-.

13. Gonzalez A, Zimbardo PG. Time in perspective; the time sense we learn early affects how we do our jobs and enjoy our pleasures. Psychol Today. 1985;19:20-

14. Holman EA, Cohen Silver R. Getting "Stuck" in the Past: Temporal Orientation and Coping With Trauma. J Pers Soc Psychol. 1998;74(5):1146-63. 
15. Webster J. A New Measure of Time perspective: Initial Psychometric Findings for the Balanced Time perspective Scale (BTPS). Can J Behav Sci Can Sci Comport. 2011 Apr 1;43:111-8.

16. Webster JD, Bohlmeijer ET, Westerhof GJ. Time to flourish: the relationship of temporal perspective to well-being and wisdom across adulthood. Aging Ment Health. 2014;18(8):1046-56.

17. van Beek W, Berghuis H, Kerkhof A, Beekman ATF. Time perspective, personality and psychopathology: Zimbardo's time perspective inventory in psychiatry. Time Soc. 2011;20(3):36474.

18. Dalley JW, Robbins TW. Fractionating impulsivity: neuropsychiatric implications. Nat Rev Neurosci. 2017;18(3):158-71.

19. Harvanko AM, Strickland JC, Slone SA, Shelton BJ, Reynolds BA. Dimensions of impulsive behavior: Predicting contingency management treatment outcomes for adolescent smokers. Addict Behav. 2019;90:334-40.

20. Bornovalova MA, Lejuez CW, Daughters SB, Zachary Rosenthal M, Lynch TR. Impulsivity as a common process across borderline personality and substance use disorders. Clin Psychol Rev. 2005 Sep;25(6):790-812.

21. Weissenberger S, Děchtěrenko F, Klicperova-Baker M, Vňuková M, Zimbardo P, Raboch J, et al. ADHD Symptoms in Adults and Time perspectives - Findings From a Czech National Sample. Front Psychol. 2020;11:950-950.

22. Henry H, Zacher H, Desmette D. Future Time perspective in the Work Context: A Systematic Review of Quantitative Studies. Front Psychol. 2017;8:413-413.

23. Kooij DTAM, Kanfer R, Betts M, Rudolph CW. Future Time perspective: A Systematic Review and Meta-Analysis. J Appl Psychol. 2018;103(8):867-93.

24. Peetsma TTD. Future Time perspective as a Predictor of School Investment. Scand J Educ Res. 2000;44(2):177-92.

25. Drake L, Duncan E, Sutherland F, Abernethy C, Henry C. Time perspective and Correlates of Wellbeing. Time Soc. 2008 Mar;17(1):47-61.

26. Stolarski M, Matthews G, Postek S, Zimbardo PG, Bitner J. How We Feel is a Matter of Time: Relationships Between Time perspectives and Mood. J Happiness Stud. 2013;15(4):809-27.

27. Stolarski M, Wiberg B, Osin E. Assessing Temporal Harmony: The Issue of a Balanced Time perspective. In: Stolarski M, Fieulaine N, van Beek W, editors. Time perspective Theory; Review, Research and Application: Essays in Honor of Philip G Zimbardo [Internet]. Cham: Springer International Publishing; 2015 [cited 2020 Nov 7]. p. 57-71. Available from: https://doi.org/10.1007/978-3-319-07368-2_3

28. Boniwell I, Zimbardo PG. Balancing Time perspective in Pursuit of Optimal Functioning. Hoboken, NJ, USA: John Wiley \& Sons, Inc; 2015. p. 223-36.

29. Sobol-Kwapinska M, Jankowski T, Przepiorka A. What do we gain by adding time perspective to mindfulness? Carpe Diem and mindfulness in a temporal framework. Personal Individ Differ. 2016 Apr;93:112-7.

Page $14 / 18$ 
30. Stolarski M, Vowinckel J, Jankowski KS, Zajenkowski M. Mind the balance, be contented: Balanced time perspective mediates the relationship between mindfulness and life satisfaction. Personal Individ Differ. 2016 Apr;93:27-31.

31. Dunkel CS, Weber JL. Using Three Levels of Personality to Predict Time perspective. Curr Psychol. 2010 Jun;29(2):95-103.

32. Weissenberger S, Klicperova-Baker M, Zimbardo P, Schonova K, Akotia D, Kostal J, et al. ADHD and Present Hedonism: time perspective as a potential diagnostic and therapeutic tool. Neuropsychiatr Dis Treat. 2016;12:2963-71.

33. Amlung M, Marsden E, Holshausen $\mathrm{K}$, Morris V, Patel H, Vedelago L, et al. Delay Discounting as a Transdiagnostic Process in Psychiatric Disorders: A Meta-analysis. JAMA Psychiatry. 2019 Nov 1;76(11):1176.

34. Oyanadel C, Buela-Casal G. Time perception and psychopathology: Influence of time perspective on quality of life of severe mental illness. Actas Esp Psiquiatr. 2014 May 1;42:99-107.

35. Fuchs T. Temporality and psychopathology. Phenomenol Cogn Sci. 2013 Mar;12(1):75-104.

36. Mostowik J, Mielimąka M, Rutkowski K, Ostrowski T. Time in the Mental Health Context: Neurotic Symptomatology, Defense Mechanisms and the Perception of Time perspective Among Patients Diagnosed With Neurotic and Personality Disorders - A Clinical Study. Psychol Rep. 2020 Dec 21;0033294120978164.

37. Rung JM, Madden GJ. Experimental reductions of delay discounting and impulsive choice: A systematic review and meta-analysis. J Exp Psychol Gen. 2018 Sep;147(9):1349-81.

38. Mostowik J, Cyranka K. New trends in psychotherapy: the role of time perspective in mental health and practiced therapeutic interventions. Psychoterapia. 2018(1 (184)).

39. Barrett K, O'Connor M, McHugh L. A Systematic Review of Values-Based Psychometric Tools Within Acceptance and Commitment Therapy (ACT). Psychol Rec. 2019 Dec;69(4):457-85.

40. Dindo L, Van Liew JR, Arch JJ. Acceptance and Commitment Therapy: A Transdiagnostic Behavioral Intervention for Mental Health and Medical Conditions. Neurotherapeutics. 2017 Jul;14(3):546-53.

41. Sword RM, Sword RKM, Brunskill SR, Zimbardo PG. Time perspective Therapy: A New Time-Based Metaphor Therapy for PTSD. J Loss Trauma. 2014;19(3):197-201.

42. Sword RM, Sword RKM, Brunskill SR. Time perspective Therapy: Transforming Zimbardo's Temporal Theory into Clinical Practice. In: Stolarski M, Fieulaine N, van Beek W, editors. Time perspective Theory; Review, Research and Application [Internet]. Cham: Springer International Publishing; 2015 [cited 2020 Nov 7]. p. 481-98. Available from: http://link.springer.com/10.1007/978-3-319-073682_31

43. Zimbardo P, Sword R, Sword R. The time cure: Overcoming PTSD with the new psychology of time perspective therapy. John Wiley \& Sons; 2012.

44. Davies S, Filippopoulos P. Changes in Psychological Time perspective During Residential Addiction Treatment: A Mixed-Methods Study. J Groups Addict Recovery. 2015 Jul 3;10(3):249-70. 
45. Mehdi Esfahani, Mohammad Bagher Kjbaf, Mohammad Reza Abedi. Evaluation and Comparison of the Effects of Time perspective Therapy, Acceptance and Commitment Therapy and Narrative Therapy on Severity of Symptoms of Obsessive-Compulsive Disorder. J Indian Acad Appl Psychol. 2015;41(3):148-

46. van Beek W, Kerkhof A, Beekman A. Future oriented group training for suicidal patients: a randomized clinical trial. BMC Psychiatry. 2009 Dec;9(1):65.

47. Kim HS (Andrew), Wohl MJA. The Bright Side of Self-Discontinuity: Feeling Disconnected With the Past Self Increases Readiness to Change Addictive Behaviors (via Nostalgia). Soc Psychol Personal Sci. 2015 Mar 1;6(2):229-37.

48. Rudolph CW, Kooij DTAM, Rauvola RS, Zacher H. Occupational future time perspective: A metaanalysis of antecedents and outcomes. J Organ Behav. 2018;39(2):229-48.

49. Rudolph CW, Kooij DTAM, Rauvola RS, Zacher H. Occupational future time perspective: A metaanalysis of antecedents and outcomes. J Organ Behav. 2018;39(2):229-48.

50. Andre L, van Vianen AEM, Peetsma TTD, Oort FJ. Motivational power of future time perspective: Meta-analyses in education, work, and health. PloS One. 2018;13(1):e0190492-e0190492.

51. Zacher $\mathrm{H}$, Frese M. Remaining time and opportunities at work: relationships between age, work characteristics, and occupational future time perspective. Psychol Aging. 2009;24(2):487.

52. Strathman A, Gleicher F, Boninger DS, Edwards CS. The consideration of future consequences: weighing immediate and distant outcomes of behavior. J Pers Soc Psychol. 1994;66(4):742.

53. Lang FR, Carstensen LL. Time counts: future time perspective, goals, and social relationships. Psychology and aging. 2002 Mar;17(1):125.

54. Hershey DA, Mowen JC. Psychological Determinants of Financial Preparedness for Retirement. The Gerontologist. 2000 Dec 1;40(6):687-97.

55. Gjesme T. Future Time Orientation as a Function of Achievement Motives, Ability, Delay of Gratification, and Sex. J Psychol. 1979 Mar;101(2):173-88.

56. Viechtbauer W. Conducting Meta-Analyses in $R$ with the metafor Package. J Stat Softw [Internet]. 2010 [cited 2020 Nov 11];36(3). Available from: http://www.jstatsoft.org/v36/i03/

\section{Figures}




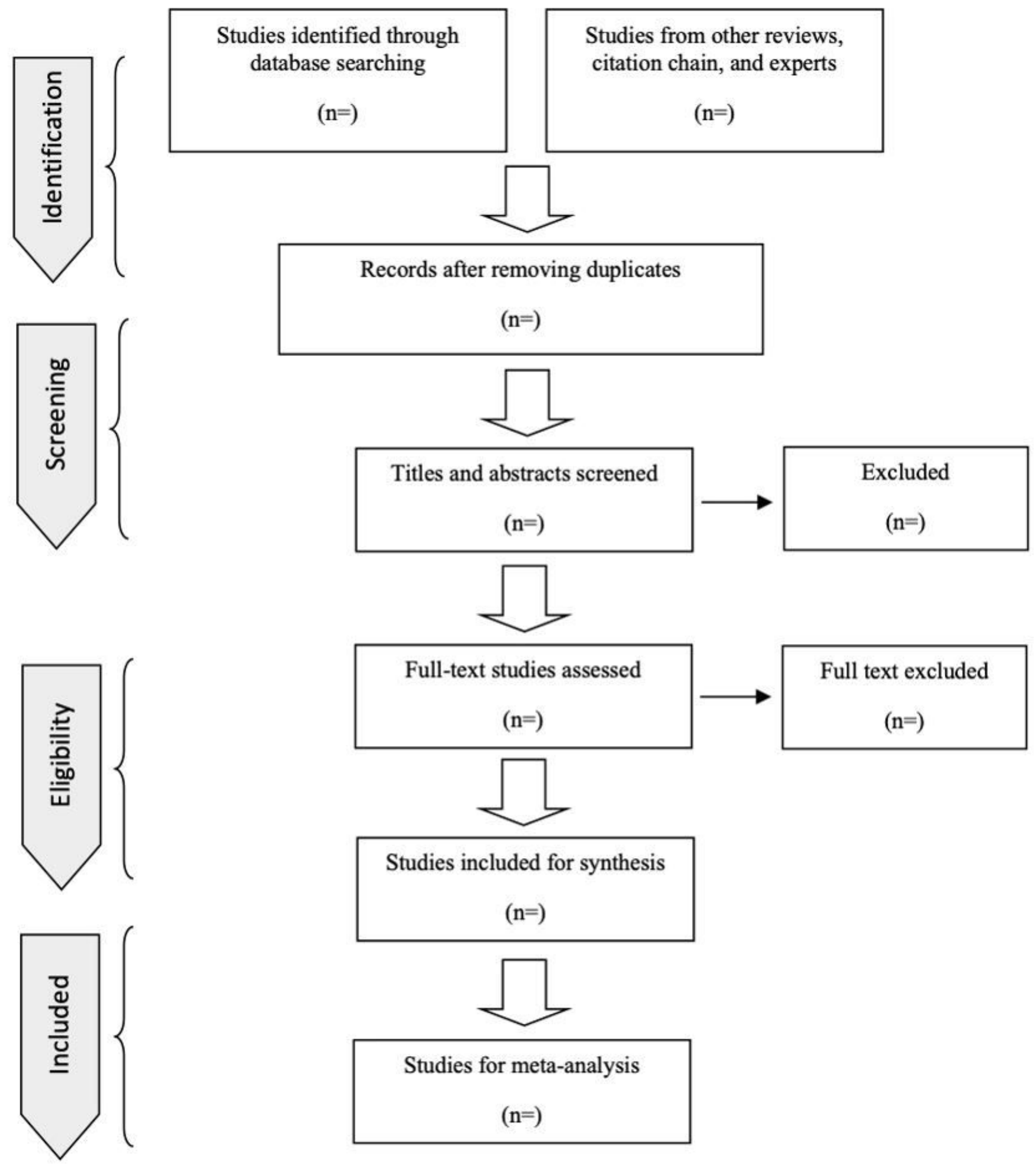

\section{Figure 1}

PRISMA flow diagram 


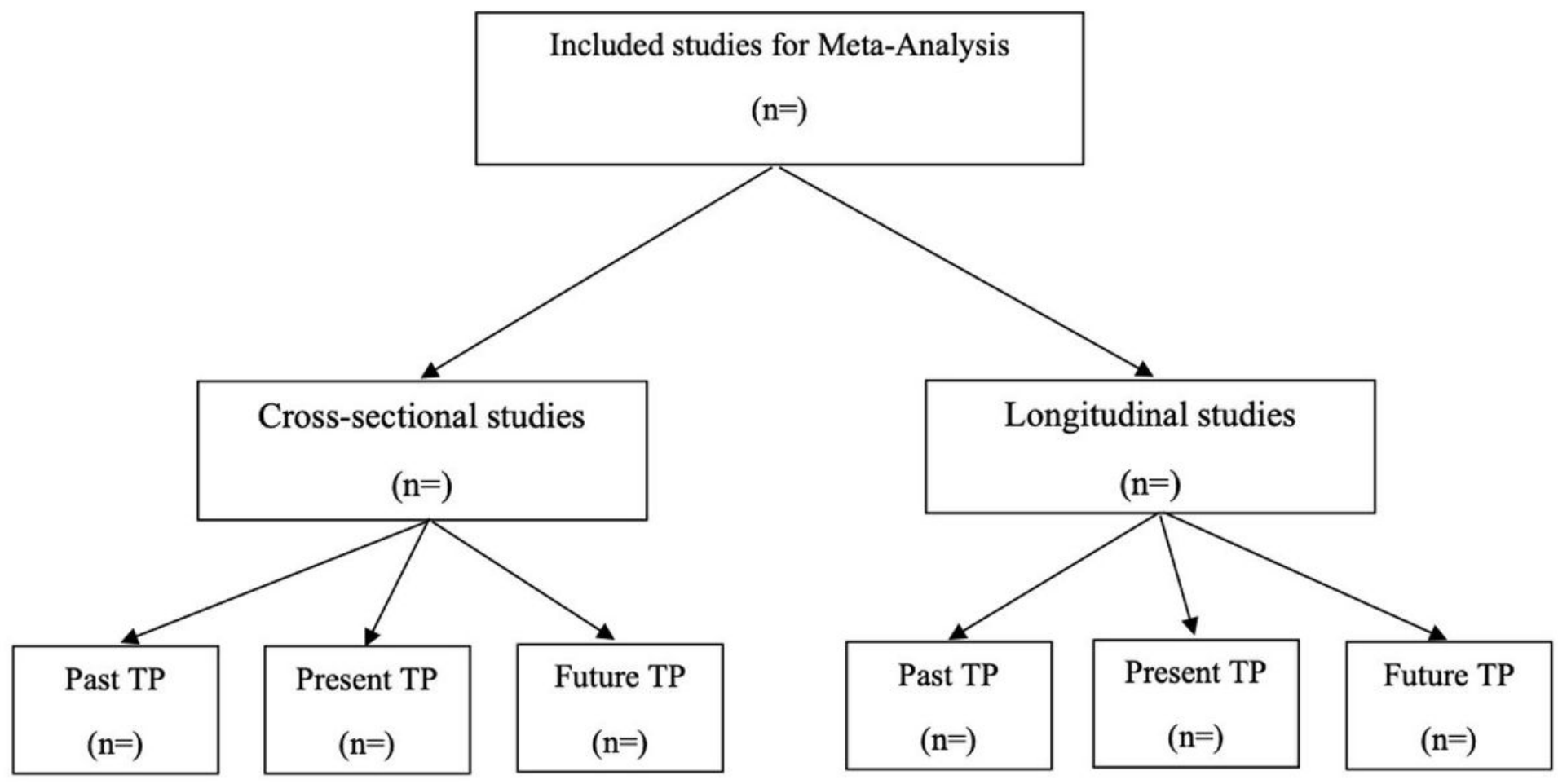

Figure 2

Meta-Analysis Stratification

Supplementary Files

This is a list of supplementary files associated with this preprint. Click to download.

- PRISMAPchecklist.docx 